\title{
Role of abemaciclib in primary breast cancer: a narrative review of MonarchE
}

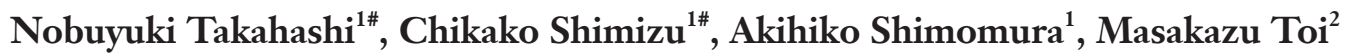 \\ ${ }^{1}$ Department of Breast and Medical Oncology, National Center for Global Health and Medicine, Tokyo, Japan; ${ }^{2}$ Breast Cancer Unit, Kyoto \\ University Hospital, Kyoto, Japan \\ Contributions: (I) Conception and design: All authors; (II) Administrative support: None; (III) Provision of study materials or patients: All authors; \\ (IV) Collection and assembly of data: All authors; (V) Data analysis and interpretation: All authors; (VI) Manuscript writing: All authors; (VII) Final \\ approval of manuscript: All authors. \\ \#These authors contributed equally to this work. \\ Correspondence to: Nobuyuki Takahashi. Department of Breast and Medical Oncology, National Center for Global Health and Medicine, Tokyo, \\ Japan. Email: nnobtakahashi@hosp.ncgm.go.jp.
}

\begin{abstract}
Estrogen affects cyclin signaling which results in proliferation of breast cancer cells. Breast cancers expressing hormone receptors (known as hormone receptor-positive or HR+ breast cancers) are characterized by dysregulation of cyclin-dependent kinase 4 and 6 (CDK4/6) activity due to overexpression and amplification of genes associated with the cell cycle. Inhibition of CDK4/6, in combination with endocrine therapy (ET), have shown significant clinical efficacy in treating HR+, human epidermal growth factor receptor 2-negative (HER2-) advanced breast cancer, leading to global approval of this combination. Abemaciclib is a CDK4/6 inhibitor with higher potency and inhibits a wider range of CDKs compared with other CDK4/6 inhibitors. The MonarchE study is a global, open-label, randomized phase III study of the efficacy of 2-year abemaciclib treatment, together with standard adjuvant ET, in patients who underwent surgery for early-stage HR+, HER2- breast cancer with anatomical or pathological high-risk recurrence features. Preplanned interim analysis of the MonarchE study showed significantly improved invasive diseasefree survival (IDFS) and distant relapse-free survival (DRFS) with the use of abemaciclib-ET combination therapy in comparison with ET alone. This review focuses on the emerging results and limitations of the MonarchE study in determining the way forward from the CDK4/6-ET combination treatment in HR+, HER2 - early-stage breast cancer.
\end{abstract}

Keywords: Abemaciclib; MonarchE; CDK4/6 inhibitors; adjuvant therapy

Received: 04 September 2021; Accepted: 21 October 2021.

doi: $10.21037 /$ tbcr-21-27

View this article at: https://dx.doi.org/10.21037/tbcr-21-27

\section{Introduction}

Abnormal cell proliferation is a hallmark of cancer (1). Cyclins and cyclin-dependent kinases (CDKs) play key roles in cell cycle regulation in proliferating cancer cells. Breast cancers expressing hormone receptors (known as hormone receptor-positive/HR+ cancers) are characterized by dysregulated CDK 4 and 6 (CDK4/6) activity due to overexpression and amplification of genes associated with the cell cycle (such as CCND1) (2). Estrogen affects the cyclin-
CDK complex via estrogen receptor signaling and results in the proliferation of breast cancer cells. These findings have led to clinical trials and global approval of combination treatment with CDK4/6 inhibitors and endocrine therapy (ET) for patients with metastatic HR+ and human epidermal growth factor receptor 2-negative (HER2-) breast cancer.

Abemaciclib is a CDK4/6 inhibitor with higher potency and inhibits a wider range of CDKs than the two other CDK4/6 inhibitors (palbociclib and ribociclib). It has been approved by the United States Food and Drug 
Administration (FDA). These CDK4/6 inhibitors were initially approved for previously treated HR+, HER2metastatic breast cancers. Subsequent studies showed that the addition of CDK4/6 inhibitors to the ET was also effective for previously untreated patients. The MonarchE study is a global randomized phase III study evaluating the efficacy of adding abemaciclib to ET for early-stage breast cancer. This study has shown promising results in terms of efficacy of the combination treatment. In this narrative review, we discuss the emerging results of the MonarchE study and future prospects of abemaciclib treatment for patients with HR+ early-breast cancer. We present the following article in accordance with the Narrative Review reporting checklist (available at https://tbcr.amegroups. com/article/view/10.21037/tbcr-21-27/rc).

\section{Pharmacology of abemaciclib}

In mammalian cells, D-type cyclins (CCND1, CCND2, and CCND3) and CDK4/6 play critical roles in entry into the cell cycle and subsequent downstream signaling pathways; these include RAS-ERK, phosphoinositide 3-kinase, and mammalian target of rapamycin signaling networks. One of the major functions of D-type cyclin-CDK4/6 complexes is to phosphorylate and inhibit the expression of retinoblastoma protein 1 (RB1), a tumor-suppressor gene required for DNA synthesis, DNA repair, and mitosis. D-type cyclins may also interact with the HR. Cyclin D1 is required for proliferation of progenitors, driven by estrogen and progesterone, in the mammary gland (3).

$\mathrm{HR}+$ human breast cancer cell lines showed increased sensitivity to CDK4/6 inhibitors (4). These preclinical studies led to clinical trials and global approval of three CDK4/6 inhibitors (palbociclib, ribociclib, and abemaciclib) in combination with ET, for patients with metastatic HR+, HER2 - breast cancer. Abemaciclib inhibits CDK4 more selectively than the other FDA-approved CDK4/6 inhibitors. This may prevent CDK4 overexpression as a mechanism of CDK4/6 inhibitor resistance (5). Moreover, abemaciclib leads to cell cycle arrest in HR+ breast cancer cells, and induces irreversible induction of senescence and apoptosis in a timeand dose-dependent manner, compared with palbociclib (6).

Abemaciclib is continuously administered twice daily at a dosage of $150 \mathrm{mg}$ when used in combination with ET. Abemaciclib is associated with more gastrointestinal (GI) adverse effects, such as diarrhea, but less hematological toxicities (particularly neutropenia) compared with the other FDA-approved CDK4/6 inhibitors. GI adverse effects are generally well-controlled by routine antiemetics, such as metoclopramide, and prophylactic administration of loperamide is often recommended. Approximately $81 \%$ of the dose of abemaciclib is eliminated in feces as metabolites; its elimination half-life is 18.3 hours, which is consistent with the twice-daily dosing schedule (7). Abemaciclib is extensively metabolized by CYP3A4 to form equipotent, active metabolites. Therefore, caution should be exercised when co-administering abemaciclib with other CYP3A4 inhibitors or inducers, and dose adjustment of abemaciclib should be considered, if necessary (7). Abemaciclib demonstrates good central nervous system penetration, with concentrations of the parent drug and active metabolites in cerebrospinal fluid comparable to unbound plasma concentrations (8). Promising intracranial antitumor efficacy is observed in patients with metastatic HR+, HER2- breast cancer (9).

\section{Clinical evidence for the efficacy of abemaciclib in metastatic HR+, HER2- breast cancer}

Abemaciclib has shown clinically significant benefits in different settings for patients with metastatic HR+ breast cancer (Table 1). A randomized double-blind phase III study (Monarch 3) showed significantly greater progressionfree survival (PFS), which was its primary endpoint, with the combination of abemaciclib and aromatase inhibitors (AIs), compared with AIs alone [median PFS: not reached vs. 14.7 months; hazard ratio (HR): 0.54; 95\% confidence interval (CI): 0.41-0.72]. In addition, the objective response rate (ORR) was higher with the combination than with AIs alone $(48.2 \%$ vs. $34.5 \%, \mathrm{P}=0.002)(10)$. In an ETpretreated setting, a global double-blind randomized phase III study (Monarch 2) investigated the efficacy of a combination of fulvestrant and abemaciclib in patients with metastatic HR+, HER2- breast cancer. The combination group experienced an improved PFS [median PFS: 16.4 vs. 9.3 months; HR: 0.55; 95\% CI: 0.45-0.68), ORR (48\% vs. 21\%), and OS (median OS: 46.7 vs. 37.3 months; HR: 0.76; 95\% CI: 0.61-0.95], compared with the group receiving fulvestrant alone $(11,12)$. Another phase III study conducted in China, Brazil, India, and South Africa showed similar PFS and ORR benefits with the combination of abemaciclib and ET compared with ET alone (median PFS: 11.5 vs. 5.6 months; HR: 0.55; 95\% CI: 0.45-0.68; ORR: $50.0 \%$ vs. $10.5 \%$ ) (13). Single-agent abemaciclib also has substantial antitumor activity in patients with metastatic $\mathrm{HR}+$ breast cancer who showed disease progression on or after ET and chemotherapy. In a single-arm phase 
Table 1 Results of clinical trials examining abemaciclib for advanced breast cancer

\begin{tabular}{|c|c|c|c|c|c|c|c|}
\hline $\begin{array}{l}\text { Study and } \\
\text { reference }\end{array}$ & ET & Setting & Participants & $\begin{array}{l}\text { Primary } \\
\text { endpoint }\end{array}$ & ORR & PFS & os \\
\hline $\begin{array}{l}\text { Monarch3 } \\
\text { (NCT02246621) } \\
\text { (10) }\end{array}$ & $\begin{array}{l}\text { Anastrozole } \\
\text { Letrozole }\end{array}$ & $\begin{array}{l}\text { - Treatment-naïve } \\
\text { ABC } \\
\text { - Relapse }>1 \text { year } \\
\text { after adjuvant ET }\end{array}$ & $\begin{array}{l}\text { Post-menopausal } \\
\text { HR+, HER2- }\end{array}$ & PFS & $\begin{array}{c}48.2 \% \text { vs. } \\
34.5 \% \\
P=0.002\end{array}$ & $\begin{array}{l}\text { NR vs. } 14.7 \text { months } \\
\text { HR }(95 \% \mathrm{Cl}): 0.54 \\
(0.41-0.72) \\
\mathrm{P}=0.00021\end{array}$ & Not reported \\
\hline $\begin{array}{l}\text { Monarchplus } \\
\text { cohort A } \\
\text { (NCT02763566) } \\
\text { (13) }\end{array}$ & $\begin{array}{l}\text { Anastrozole } \\
\text { Letrozole }\end{array}$ & $\begin{array}{l}\text { - Treatment-naïve } \mathrm{ABC} \\
\text { - Relapse }>1 \text { year } \\
\text { after adjuvant ET } \\
\text { - Relapse }<1 \text { year } \\
\text { after adjuvant ET } \\
\text { except anastrozole or } \\
\text { letrozole }\end{array}$ & $\begin{array}{l}\text { Post-menopausal } \\
\text { HR+, HER2- }\end{array}$ & PFS & $\begin{array}{c}65.9 \% \text { vs. } \\
36.1 \% \\
P<0.0001\end{array}$ & $\begin{array}{l}\text { NR vs. } 14.7 \text { months } \\
\text { HR (95\% Cl): } 0.50 \\
(0.35-0.72) \\
P=0.0001\end{array}$ & Not reported \\
\hline $\begin{array}{l}\text { Monarchplus } \\
\text { cohort B } \\
\text { (NCT02763566) } \\
\text { (13) }\end{array}$ & Fulvestrant & $\begin{array}{l}-\leq 1 \text { line of ET and no } \\
\text { chemotherapy for ABC } \\
\text { - PD while or } \\
\leq 12 \text { months after } \\
\text { adjuvant ET }\end{array}$ & $\begin{array}{l}\text { Post-menopausal } \\
\text { HR+, HER2- }\end{array}$ & PFS & $\begin{array}{c}50.0 \% \text { vs. } \\
10.5 \% \\
P<0.0001\end{array}$ & $\begin{array}{l}11.5 \text { vs. } 5.6 \text { months } \\
\text { HR }(95 \% \mathrm{Cl}): 0.38 \\
(0.24-0.59) \\
\mathrm{P}<0.0001\end{array}$ & Not reported \\
\hline $\begin{array}{l}\text { Monarch1 } \\
\text { (NCT02102490) } \\
(14)\end{array}$ & None & $\begin{array}{l}- \text { PD on ET and } \\
\geq \text { two chemotherapy }\end{array}$ & $\begin{array}{l}\text { Pre-/post- } \\
\text { menopausal } \\
\text { HR+, HER2- }\end{array}$ & ORR & $19.7 \%$ & 6.0 months & 17.7 months \\
\hline
\end{tabular}

ET, endocrine therapy; ABC, advanced breast cancer; PD, progressive disease; HR+, hormonal receptor positive; HER2, Human Epidermal Growth Factor Receptor 2; ORR, objective response rate; PFS, progression free survival; NR, not reached; HR, hazard ratio; CI, confidence interval; OS, overall survival.

II open-label study, Monarch 1, abemaciclib showed an ORR of $20 \%$ with a median PFS of 6.0 months (14). Most abemaciclib trials have been limited to postmenopausal women or included premenopausal women with ovarian function suppression (OFS) as a small subset population. Preliminary analysis of the pre- and perimenopausal subset of patients enrolled in the Monarch 2 study showed that the addition of abemaciclib to fulvestrant and a gonadotropinreleasing hormone agonist (GnRHa) improved both PFS (not reached vs. 10.5 months; HR: 0.45; 95\% CI: 0.26-0.75) and ORR (61\% vs. 29\%), compared with those receiving fulvestrant and a GnRHa only (6).

\section{Adjuvant abemaciclib with ET: results and limitations of the MonarchE study}

As described above, abemaciclib has shown clinical benefits in metastatic HR+, HER2- breast cancer. However, more than $90 \%$ of breast cancers are diagnosed at an early stage and $70 \%$ of these cancers are HR+ (15). Adjuvant ET is associated with a significant reduction in the risk of recurrence and death $(16,17)$. However, subsets of these patients, especially those with high-risk clinical and pathological features, experience disease recurrence, often with distant metastases (18).

Recently, a preplanned interim analysis of the results of MonarchE was published (19). MonarchE is a global, openlabel, randomized phase III study evaluating the efficacy of 2-year abemaciclib treatment, in addition to standard ET, in patients who underwent surgery for early-stage breast cancer and who were categorized as having a high risk of recurrence. Adjuvant radiation and/or adjuvant or neoadjuvant chemotherapy were allowed but not required. High-risk clinical and/or pathological features were defined 
as involvement of four or more lymph nodes, involvement of 1-3 lymph nodes as well as tumor size of $\geq 5 \mathrm{~cm}$, histological grade 3, or centrally evaluated Ki-67 of $\geq 20 \%$. Exclusion criteria included patients who had previously received ET for breast cancer prevention and/or a CDK4/6 inhibitor. Those diagnosed with inflammatory breast cancer and those with a history of venous thromboembolic events (VTEs) were also excluded concerning a potential risk of VTEs with abemaciclib (20). Patients who met the inclusion criteria and unmet exclusion criteria were randomly assigned (1:1) to receive either abemaciclib $(150 \mathrm{mg}$ twice daily on a continuous dosing schedule) and ET, or ET alone. Stratification factors of randomization included previous chemotherapy (neoadjuvant, adjuvant, or none), menopausal status at the time of breast cancer diagnosis (premenopausal vs. postmenopausal), and region (North America/Europe, Asia, or others). Patients received the assigned treatment for 2 years, followed by ET alone for 5 to 10 years as clinically indicated. The primary endpoint was invasive disease-free survival (IDFS) in the intent-totreat population. From July 2017 to August 2019, 5,637 patients from 603 sites in 38 countries were enrolled in the study. Patients were predominantly postmenopausal (56.5\%) and were eligible based on $\geq 4$ lymph node involvement (59.6\%). AIs were prescribed as the first ET on study treatment in $68.3 \%$ of patients (including $14.2 \%$ treated with OFS in addition) and tamoxifen in $31.4 \%$ (including $7.6 \%$ treated with OFS in addition). In the median followup time of 15.5 months, abemaciclib together with ET demonstrated a significant improvement in IDFS compared with that in ET alone (HR, 0.75; 95\% CI: 0.60-0.93) with a 2-year IDFS of $92.2 \%$ vs. $88.7 \%$. Distant relapse-free survival (DRFS) also improved with the combination (HR: 0.72 ; $95 \%$ CI, 0.56-0.92), with a 2 -year DRFS of $93.6 \%$ vs. $90.3 \%$. These clinically insightful IDFS and DRFS results were also seen in multiple subgroups of patients who received neoadjuvant chemotherapy (21); whose highrisk feature were defined by $\mathrm{Ki}-67$ of $\geq 20 \%$ (22); and in Asian population $(23,24)$. OS data were immature. Grade $\geq 3$ adverse events (AEs) were observed in $45.9 \%$ of patients in the abemaciclib arm and in $12.9 \%$ of patients in the control arm. The most frequent grade 3 or 4 AEs in the abemaciclib arm were diarrhea (7.6\%), neutropenia (18.6\%), and fatigue $(2.8 \%)$. Abemaciclib dose adjustments due to AEs occurred in $68.1 \%$ of patients; $16.6 \%$ of patients discontinued abemaciclib because of AEs; and $6.2 \%$ of patients discontinued both. These discontinuation rates were higher than in the control arm, in which $0.8 \%$ of patients discontinued ET. Higher incidences of VTEs and interstitial lung disease (ILD) occurred in the abemaciclib arm than in the control arm $(2.7 \% v s .0 .3 \% ; 1.2 \%$ vs. one patient, respectively). The risk of VTE was even higher in patients who received tamoxifen and in patients who had a body mass index $>25$ (6). ILD was observed more frequently in the Asian population (6.6\%) (6).

As described above, preplanned analysis of the MonarchE study showed significantly greater IDFS and DRFS with the use of abemaciclib-ET combination treatment than with ET alone. Despite these promising results, several limitations must be considered. The results obtained for the combination of abemaciclib and ET are inconsistent with the results of two studies in which a combination of palbociclib and ET was evaluated. The PALLAS study was an open-label randomized phase III trial to determine if the addition of 2-year palbociclib treatment (125 $\mathrm{mg}$ once daily on days 1-21 of a 28-day cycle) to standard adjuvant ET improves IDFS in patients with early-stage HR+, HER2breast cancer. Patients with stage II to III breast cancers were eligible, in contrast with the high-risk inclusion criteria of the MonarchE study described above. In the follow-up period of 23.7 months, pre-specified interim analysis did not show significant improvement in 3-year IDFS $(88.2 \%$ in the palbociclib group $v s .88 .5 \%$ in the control group; HR: 0.93 ; $95 \%$ CI: $0.76-1.15$ ) as well as in 3 -year DRFS (89.3\% in the palbociclib group vs. $90.7 \%$ in the control group; HR: 1.00; 95\% CI: 0.79-1.27) (25). The discrepancy in efficacies of combination CDK4/6 inhibitorET treatment between the MonarchE and PALLAS studies may be due to several reasons. First, the MonarchE study included patients with a higher risk of recurrence than the PALLAS study, especially those with $\geq 4$ lymph node involvement. These patients may be better candidates for CDK4/6 inhibitor-ET combination treatment, and this contributed to the substantially greater efficacy observed in the MonarchE study. Second, AE profiles differ between abemaciclib and palbociclib (26). In the two studies, the discontinuation rate due to $\mathrm{AEs}$ was lower with abemaciclib than with palbociclib (16.6\% and $27.1 \%$, respectively). In the PALLAS study, only $32.3 \%$ of patients completed treatment and $25.5 \%$ received planned protocol therapy, suggesting a lack of adequate drug exposure. The majority of patients enrolled in the MonarchE study had continued to receive abemaciclib at the cutoff date: $12.5 \%$ completed and $72.8 \%$ continued to receive planned protocol therapy. A longer follow-up of the MonarchE study is warranted. Third, abemaciclib is more potent than palbociclib, and 
can inhibit other CDKs in addition to CDK4/6. This eventually results in the inhibition of $\mathrm{G} 1$ to $\mathrm{S}$ and $\mathrm{G} 2$ to $\mathrm{M}$ phase transitions in the cell cycle (27). Further clinical and preclinical studies directly comparing different CDK4/6 inhibitors are needed to reveal the discrepancies between the results of the two trials.

In the MonarchE study, the 2-year duration of abemaciclib treatment was chosen based on historical studies, which reported that recurrence events first peaked at 2 years in patients with early breast cancer treated with adjuvant ET (28). However, more than half of recurrences also occurred after 5 -year treatment with ET (29). Although results of later follow-ups (median follow-up duration: 19 months) of MonarchE study continuously showed benefits of IDFS and DRFS (30), with a consistent safety profile $(30,31)$, it is important to confirm results at the prespecified, final cutoff time point (3 years). Results as well as long-term toxicity should be confirmed at an even longer follow-up duration, given the usage of the combination treatment in the adjuvant setting, where many patients will survive for years without any recurrences. Both the MonarchE and PALLAS studies were open-label and unblinded. The different toxicity profiles of abemaciclib and ET may have affected the blindness of the trials. The openlabel design of these studies with the primary endpoint of IDFS evaluated by imaging performed by investigators, can cause lead-time bias, as discussed previously (32).

Another randomized, double-blind, placebo-controlled phase III study, Penelope-B, also did not show any benefit from adding palbociclib, as an adjuvant treatment, to ET for patients with HR+, HER2 - early breast cancer who received neoadjuvant chemotherapy and had residual highrisk features (33). Patients with residual high-risk features [defined by CPS+EG score (a score integrating clinical and pathological stage, estrogen receptor expression, and nuclear grade) $\geq 3$ or 2 and ypN+] after neoadjuvant chemotherapy and surgery, were randomly assigned to the 1-year treatment with ET and palbociclib group, or to the ET and placebo group. The primary endpoint was IDFS. Results showed that IDFS was not significantly different between the two groups (HR: 0.93; 95\% CI: 0.74-1.17) after a median follow-up of 42.8 months. Up to $40 \%$ of patients enrolled in the MonarchE study also received neoadjuvant chemotherapy. However, association between response to neoadjuvant chemotherapy (whether patients achieved pathological complete responses or not) and efficacy of adjuvant abemaciclib-ET combination remains to be addressed. Pre-specified subgroup analysis of
MonarchE showed that patients who received neoadjuvant chemotherapy demonstrated better improvement of IDFS and DRFS by abemaciclib-ET combination (HR: 0.614; 95\% CI: $0.473-0.797$ and HR: 0.609; 95\% CI: 0.459 0.809 , respectively) compared with the intent-to-treat population (21). The discrepancy between results of Penelope-B and MonarchE may also be due to differences in high-risk feature definitions, drugs, and/or treatment durations (34).

In the MonarchE study, high-risk of recurrence was defined by a combination of anatomical (number of lymph nodes involved and tumor size) and pathological features (histologic grade and Ki-67). In the PALLAS study, patients with stage II or III were enrolled. In the Penelope-B study, patients with a CPS+EG score $\geq 3$ or 2 with $y p N 1+$ were defined as having a high risk of recurrence. It is important to include both anatomical and pathological features to define high-risk recurrence, as shown by the MonarchE study. However, in the MonarchE study, the majority of patients were defined as high-risk based on involvement of $\geq 4$ lymph nodes. According to results of the SWOG S1007 (RxPONDER) trial, adjuvant chemotherapy had benefit to postmenopausal patients, regardless of the number of metastatic lymph nodes (35). It is, therefore, necessary to reconsider how high-risk of recurrence is defined.

We also need to consider how the results of MonarchE should be applied to the premenopausal population. In the interim analysis of the MonarchE study, patients were predominantly postmenopausal and were treated with AIs as the adjuvant ET, with only $31 \%$ and $14 \%$ of enrolled patients being treated with tamoxifen and OFS, respectively. Although a significant $37 \%$ risk reduction of IDFS with abemaciclib and ET was reported in premenopausal patients enrolled in the MonarchE study (19), further investigations are needed to confirm these results. A higher incidence of VTEs was observed in the abemaciclib and ET arm, particularly in combination with tamoxifen (6). The choice of ET should be made carefully and is a subject warranting validation in the future.

\section{Perspective: what is the way forward from MonarchE?}

Patients enrolled in MonarchE are conceptually divided into three groups: (I) patients with poor prognosis and inadequate response to the addition of abemaciclib; (II) patients who are highly sensitive to ET and have no benefit with the addition of abemaciclib; and (III) patients having 
a high risk of recurrence, for whom the recurrence risk is reduced with the addition of abemaciclib to ET (34). Identification of the third group is necessary to improve the efficacy of abemaciclib-ET combination treatment and to avoid overtreatment of patients who do not need it. There are several potential indicators to identify patients who may benefit from combination treatment. As described above, subgroup analysis of patients who received neoadjuvant chemotherapy showed greater improvement of IDFS and DRFS compared with the intent-to-treat population (21), although these improvements in the outcomes need to be cautiously compared. The high-risk of recurrence feature in the majority of patients enrolled in the MonarchE study was defined as involvement of $\geq 4$ lymph nodes. It is important to evaluate the efficacy with abemaciclibET combination particularly in such population. Previous studies have suggested that several genomic characteristics, such as CDK6 amplification and polyclonal RB1 mutation, can predict CDK4/6 inhibitor resistance (36,37). Currently, these genomic biomarker-based hypotheses have been tested in metastatic breast cancer in multiple studies (NCT03130439, NCT04432454, NCT04256941, and NCT04964934). Exploratory studies using resected tumors are needed to identify these biomarkers to predict which patients will be more likely to benefit from combination treatment in the adjuvant setting. Translational research in MonarchE and PALLAS is ongoing to identify predictive biomarkers. Surgically resected tumors will be suitable for such studies and will guide us in answering these important questions. In addition, the duration of adjuvant CDK4/6 inhibitors should further be discussed.

As the CDK4/6 inhibition alone may not be sufficient to achieve antitumor efficacy, it is also important to predict ET sensitivity. In the Monarch 2 trial, longer OS was observed with an abemaciclib-fulvestrant combination, compared with fulvestrant alone, in both the previously ETsensitive subgroup (known as the "secondary ET resistance" group; median OS: 48.8 vs. 40.7 months) and the ETresistant subgroup (known as the "primary ET resistance" group; median OS: 38.7 vs. 31.5 months) (12). In contrast, according to a subgroup analysis in the PALOMA-3 trial, a double-blind randomized phase III study of palbociclibfulvestrant combination for metastatic HR+, HER2- breast cancer, the OS was numerically shorter in the palbociclib group than in the placebo group in patients with intrinsic resistance to prior ET (median OS: 20.2 vs. 26.2 months). OS was significantly longer, however, in the ET-sensitive population (39.7 vs. 29.7 months; HR: 0.72; 95\% CI:
0.55-0.94) (38). Several genomic characteristics including ESR1 alteration and epigenetic changes are associated with ET resistance (39). Resistance mechanisms of ET should be addressed, along with addressing CDK4/6 inhibitors sensitivity for ET-resistant population, in studies exploring results of the MonarchE study.

According to the National Comprehensive Cancer Network guidelines (40), patients with early breast cancer with $\geq 4$ regional lymph nodes involved (pN2 or N3), or 1-3 lymph nodes involved with a high 21-gene reverse transcriptase polymerase chain reaction assay score $(\geq 26)$, and who have not received neoadjuvant chemotherapy, should consider adjuvant chemotherapy. These patients may also meet the inclusion criteria of the MonarchE study. Future studies must address the role of adjuvant chemotherapy as well as potential additional toxicities of abemaciclib-ET combination treatment when patients have already received adjuvant chemotherapy. Although two-thirds of patients received adjuvant chemotherapy in the MonarchE study, efficacy and toxicities with the abemaciclib-ET combination remain to be addressed within this population.

Using the abemaciclib-ET combination for treatment in neoadjuvant settings also needs to be investigated by future studies. The NeoMonarch study showed a significant reduction in pathological Ki-67 with the addition of abemaciclib to anastrozole in the neoadjuvant setting, compared with anastrozole alone (41). A similar improvement was also reported with palbociclib in the PALLET study (42). However, the efficacy of ET over chemotherapy in the neoadjuvant setting remains controversial in both premenopausal and postmenopausal patients with HR+, HER2 - early breast cancer $(43,44)$. In the era of CDK4/6 inhibitor-ET combination treatment for HR+, HER2- breast cancer, it is important to revisit which treatment option, the CDK4/6 inhibitor-ET combination or standard chemotherapy, has better efficacy in the neoadjuvant or adjuvant setting for patients with $\mathrm{HR}+$, HER2 + early breast cancer. The efficacy of peri-operative abemaciclib against early breast cancer is being tested in several clinical trials (Table 2). The questions mentioned above need to be addressed in these studies.

Going forward, the possibility of adding a third agent to the abemaciclib-ET treatment combination needs to be investigated. Numerous preclinical and emerging clinical studies have shown promising synergistic efficacy with CDK4/6 inhibitors by targeting multiple biological pathways. These include agents targeting HER2, 
Table 2 Clinical trials of abemaciclib for early breast cancer

\begin{tabular}{|c|c|c|c|c|c|c|c|}
\hline $\begin{array}{l}\text { NCT identifier } \\
\text { trial name }\end{array}$ & Phase & Setting & Investigational Tx & Comparison & Participants & $\begin{array}{l}\text { Primary } \\
\text { endpoint }\end{array}$ & Status \\
\hline $\begin{array}{l}\text { NCT03155997 } \\
\text { MonarchE }\end{array}$ & 3 & Adjuvant & $\begin{array}{l}\text { Abemaciclib and ET } \\
\text { (investigator's choice) }\end{array}$ & ET alone & $\begin{array}{l}\text { - } \text { Pre-/post-menopausal } \\
\text { - HR+, HER2- EBC } \\
\text { - }>4 \text { axillary LN OR } 1-3 \text { LN with } \\
\text { tumor size } \geq 5 \mathrm{~cm} \text { or histologic } \\
\text { grade } 3 \text { or Ki67 } \geq 20 \%\end{array}$ & IDFS & $\begin{array}{l}\text { Active not } \\
\text { recruiting }\end{array}$ \\
\hline $\begin{array}{l}\text { NCT04565054 } \\
\text { ADAPTlate }\end{array}$ & 3 & Adjuvant & $\begin{array}{l}\text { Abemaciclib and ET } \\
\text { (investigator's choice) }\end{array}$ & ET alone & $\begin{array}{l}\text { - Pre-/post-menopausal } \\
\text { - HR+, HER2- EBC } \\
\text { - After completion of 2-6 years } \\
\text { of ET } \\
\text { Clinical high-risk features } \\
\text { (Axillary LN involvement, } \\
\text { high recurrence score, high } \\
\text { histologic grade, non-pCR with } \\
\text { neoadjuvant therapy) }\end{array}$ & IDFS & Recruiting \\
\hline $\begin{array}{l}\text { NCT04584853 } \\
\text { POETIC-A }\end{array}$ & 3 & Neoadjuvant & $\begin{array}{l}\text { Abemaciclib and ET } \\
\text { (investigator's choice) }\end{array}$ & ET alone & $\begin{array}{l}\text { Post-menopausal HR+, } \\
\text { HER2- EBC } \\
\text { Ki67 } \geq 20 \% \text { OR histologic grade } \\
3 \text { OR tumor size }>5 \mathrm{~cm} \text { OR PgR } \\
\text { negative OR PgR unknown and } \\
\text { vascular invasion }\end{array}$ & TTR & Recruiting \\
\hline $\begin{array}{l}\text { NCT04293393 } \\
\text { CARABELA }\end{array}$ & 2 & Neoadjuvant & $\begin{array}{l}\text { Abemaciclib and } \\
\text { letrozole }\end{array}$ & $\begin{array}{l}\text { ChemoTx } \\
(\mathrm{AC}->\mathrm{T})\end{array}$ & $\begin{array}{l}\text { - } \text { Pre-/post-menopausal } \\
\text { - HR+, HER2- EBC } \\
\text { - cStage II-III OR T2N0 with } \\
\text { Ki67 >30\% OR T2N0 with Ki67 } \\
\text { of } 20-30 \% \text { and PgR- and/or } \\
\text { histological grade } 3\end{array}$ & $\begin{array}{l}\text { Residual } \\
\text { cancer } \\
\text { burden } \\
0 \text {-I rate }\end{array}$ & Recruiting \\
\hline NCT04305236 & 2 & Neoadjuvant & $\begin{array}{l}\text { Abemaciclib and } \\
\text { fulvestrant }\end{array}$ & $\begin{array}{l}\text { None } \\
\text { (single-arm) }\end{array}$ & $\begin{array}{l}\text { - Post-menopausal } \\
\text { - HR+ EBC, HER2 irrespective } \\
\text { - cStage I-III }\end{array}$ & pCR rate & Recruiting \\
\hline $\begin{array}{l}\text { NCT02831530 } \\
\text { ABC-POP }\end{array}$ & 2 & Neoadjuvant & Abemaciclib & $\begin{array}{l}\text { None } \\
\text { (single-arm) }\end{array}$ & $\begin{array}{l}\text { - Pre-/post-menopausal } \\
\text { - HR+ EBC, HER2 irrespective }\end{array}$ & $\begin{array}{l}\text { Ki67 } \\
\text { change }\end{array}$ & $\begin{array}{l}\text { Completed no } \\
\text { results posted }\end{array}$ \\
\hline
\end{tabular}

Table 2 (continued) 
Table 2 (continued)

\begin{tabular}{|c|c|c|c|c|c|c|c|}
\hline $\begin{array}{l}\text { NCT identifier } \\
\text { trial name }\end{array}$ & Phase & Setting & Investigational Tx & Comparison & Participants & $\begin{array}{l}\text { Primary } \\
\text { endpoint }\end{array}$ & Status \\
\hline $\begin{array}{l}\text { NCT03979508 } \\
\text { BEAUTY }\end{array}$ & 2 & Neoadjuvant & Abemaciclib & Surgery alone & $\begin{array}{l}\text { - Triple negative breast cancer } \\
\text { - Stage I-III }\end{array}$ & $\begin{array}{l}\text { CD8/ } \\
\text { FOXP3 } \\
\text { change in } \\
\text { tumor }\end{array}$ & Recruiting \\
\hline NCT04088032 & 1 & Neoadjuvant & $\begin{array}{l}\text { Abemaciclib and } \\
\text { durvalumab and } \mathrm{Al}\end{array}$ & $\begin{array}{l}\text { None (single- } \\
\text { arm) }\end{array}$ & $\begin{array}{l}\text { - Post-menopausal } \\
\text { - HR+, HER2- EBC } \\
\text { - cStage II-III }\end{array}$ & Safety & $\begin{array}{l}\text { Withdrawn } \\
\text { (Per sponsor) }\end{array}$ \\
\hline
\end{tabular}

Status of clinical trials were accessed on 29th September 2021 in clinicaltrial.gov https://clinicaltrials.gov/. NCT, National Clinical Trial; Tx, treatment; ET, endocrine therapy; HR, hormone receptor; HER2, Human Epidermal Growth Factor Receptor 2; EBC, early breast cancer; LN, lymph node; IDFS, invasive disease free survival; $\mathrm{PCR}$, pathological complete response; PgR, progesterone receptor; TTR, time to tumor recurrence; cStage, clinical stage; ChemoTx (AC -> T), chemotherapy with doxorubicin and cyclophosphamide followed by taxane; $\mathrm{Al}$, aromatase inhibitor.

phosphoinositide 3-kinase, mammalian target of rapamycin signaling, protein kinase $\mathrm{B}$, fibroblast growth factor receptor, and poly (ADP-ribose) polymerases (PARP) (45). Recently, the adjuvant PARP inhibitor olaparib showed significant benefits of IDFS and OS for patients with HER2 - early breast cancer with BRCA1 or BRCA2 germline pathogenic or likely pathogenic variants (46). Looking forward, the way in which these populations can be treated with the abemaciclib-ET combination must be considered.

\section{Conclusions}

In conclusion, although pre-specified interim analyses of the MonarchE study have shown the promising results of abemaciclib-ET combination treatment, there are still multiple clinical questions to be addressed. Results of studies addressing these questions as well as exploratory studies of MonarchE will provide guidance on the way forward in the era of CDK4/6 inhibitor-ET combination treatment for patients with HR+, HER2- breast cancer.

\section{Acknowledgments}

Funding: None.

\section{Footnote}

Reporting Checklist: The authors have completed the Narrative Review reporting checklist. Available at https:// tbcr.amegroups.com/article/view/10.21037/tbcr-21-27/rc

Conflicts of Interest: All authors have completed the ICMJE uniform disclosure form (available at https://tbcr. amegroups.com/article/view/10.21037/tbcr-21-27/coif). CS reports that he is a Steering Committee member of MonarchE and receives a research grant from Eli-Lilly. AS reports that he receives research grant from Chugai Pharmaceutical, AstraZeneca, Daiichi Sankyo, Taiho Pharmaceutical, Mochida Pharmaceutical, Eisai, and serves as a lecture honoraria or lecture chair held by Chugai Pharmaceutical, AstraZeneca, Daiichi Sankyo, Eisai, Eli Lilly, Pfizer, Kyowa Kirin, Novartis, Takeda, MSD. MT reports that he receives research grants from Chugai, Takeda, Pfizer, Kyowa-Kirin, Taiho, JBCRG assoc., Eisai, Eli Lilly, Daiichi-Sankyo, AstraZeneca, Astellas, Shimadzu, Yakult, Nippon Kayaku, AFI technology, Luxonus, Shionogi, GL Science, serves as a lecture honoraria or lecture chair held by Chugai, Takeda, Pfizer, KyowaKirin, Taiho, Eisai, Daiichi-Sankyo, AstraZeneca, Eli Lilly, MSD, Exact Science, Novartis, Konica Minolta, Shimadzu, 
Yakult, Nippon Kayaku, is an advisory board of KyowaKirin, Daiichi-Sankyo, Eli Lilly, Konica Minolta, BMS, Athenex Oncology, Bertis, Terumo, Kansai Medical Net, is a member of the board of directors (no salary) of JBCRG assoc., KBCRN, OOTR, is an associate editor of Scientific Reports, Breast Cancer Research and Treatment, Cancer Science, Frontiers in Women's Cancer, Asian fournal of Surgery, Asian Fournal of Breast Surgery, and is a deputy editor of International Fournal of Oncology. NT has no conflicts of interest to declare.

Ethical Statement: The authors are accountable for all aspects of the work in ensuring that questions related to the accuracy or integrity of any part of the work are appropriately investigated and resolved.

Open Access Statement: This is an Open Access article distributed in accordance with the Creative Commons Attribution-NonCommercial-NoDerivs 4.0 International License (CC BY-NC-ND 4.0), which permits the noncommercial replication and distribution of the article with the strict proviso that no changes or edits are made and the original work is properly cited (including links to both the formal publication through the relevant DOI and the license). See: https://creativecommons.org/licenses/by-nc-nd/4.0/.

\section{References}

1. Hanahan D, Weinberg RA. Hallmarks of cancer: the next generation. Cell 2011;144:646-74.

2. Arnold A, Papanikolaou A. Cyclin D1 in breast cancer pathogenesis. J Clin Oncol 2005;23:4215-24.

3. Fantl V, Stamp G, Andrews A, et al. Mice lacking cyclin D1 are small and show defects in eye and mammary gland development. Genes Dev 1995;9:2364-72.

4. Finn RS, Dering J, Conklin D, et al. PD 0332991, a selective cyclin D kinase 4/6 inhibitor, preferentially inhibits proliferation of luminal estrogen receptor-positive human breast cancer cell lines in vitro. Breast Cancer Res 2009;11:R77.

5. Martin LA, Pancholi S, Ribas R, et al. Abstract P3-03-09: Resistance to palbociclib depends on multiple targetable mechanisms highlighting the potential of drug holidays and drug switching to improve therapeutic outcome. Cancer Res 2017. doi: 10.1158/1538-7445.SABCS16-P3-03-09

6. Toi M, Harbeck N, Puig JM, et al. 44O Characterization of venous thromboembolic events (VTE), elevated aminotransferases (EAT) and interstitial lung disease (ILD) in monarchE. Ann Oncol 2021;32:S39-40.

7. Fogli S, Del Re M, Curigliano G, et al. Drug-drug interactions in breast cancer patients treated with CDK4/6 inhibitors. Cancer Treat Rev 2019;74:21-8.

8. Raub TJ, Wishart GN, Kulanthaivel P, et al. Brain Exposure of Two Selective Dual CDK4 and CDK6 Inhibitors and the Antitumor Activity of CDK4 and CDK6 Inhibition in Combination with Temozolomide in an Intracranial Glioblastoma Xenograft. Drug Metab Dispos 2015;43:1360-71.

9. Tolaney SM, Sahebjam S, Le Rhun E, et al. A Phase II Study of Abemaciclib in Patients with Brain Metastases Secondary to Hormone Receptor-Positive Breast Cancer. Clin Cancer Res 2020;26:5310-9.

10. Goetz MP, Toi M, Campone M, et al. MONARCH 3: Abemaciclib As Initial Therapy for Advanced Breast Cancer. J Clin Oncol 2017;35:3638-46.

11. Sledge GW Jr, Toi M, Neven P, et al. MONARCH 2: Abemaciclib in Combination With Fulvestrant in Women With HR+/HER2- Advanced Breast Cancer Who Had Progressed While Receiving Endocrine Therapy. J Clin Oncol 2017;35:2875-84.

12. Sledge GW Jr, Toi M, Neven P, et al. The Effect of Abemaciclib Plus Fulvestrant on Overall Survival in Hormone Receptor-Positive, ERBB2-Negative Breast Cancer That Progressed on Endocrine TherapyMONARCH 2: A Randomized Clinical Trial. JAMA Oncol 2020;6:116-24.

13. Zhang QY, Sun T, Yin YM, et al. MONARCH plus: abemaciclib plus endocrine therapy in women with HR+/HER2- advanced breast cancer: the multinational randomized phase III study. Ther Adv Med Oncol 2020;12:1758835920963925.

14. Dickler MN, Tolaney SM, Rugo HS, et al. MONARCH 1, A Phase II Study of Abemaciclib, a CDK4 and CDK6 Inhibitor, as a Single Agent, in Patients with Refractory HR+/HER2- Metastatic Breast Cancer. Clin Cancer Res 2017;23:5218-24.

15. Cardoso F, Spence D, Mertz S, et al. Global analysis of advanced/metastatic breast cancer: Decade report (20052015). Breast 2018;39:131-8.

16. Early Breast Cancer Trialists' Collaborative Group (EBCTCG); Davies C, Godwin J, et al. Relevance of breast cancer hormone receptors and other factors to the efficacy of adjuvant tamoxifen: patient-level meta-analysis of randomised trials. Lancet 2011;378:771-84.

17. Howell A, Cuzick J, Baum M, et al. Results of the ATAC (Arimidex, Tamoxifen, Alone or in Combination) trial 
after completion of 5 years' adjuvant treatment for breast cancer. Lancet 2005;365:60-2.

18. Early Breast Cancer Trialists' Collaborative Group (EBCTCG). Aromatase inhibitors versus tamoxifen in early breast cancer: patient-level meta-analysis of the randomised trials. Lancet 2015;386:1341-52.

19. Johnston SRD, Harbeck N, Hegg R, et al. Abemaciclib Combined With Endocrine Therapy for the Adjuvant Treatment of HR+, HER2-, Node-Positive, HighRisk, Early Breast Cancer (monarchE). J Clin Oncol 2020;38:3987-98.

20. Rugo HS, Huober J, García-Sáenz JA, et al. Management of Abemaciclib-Associated Adverse Events in Patients with Hormone Receptor-Positive, Human Epidermal Growth Factor Receptor 2-Negative Advanced Breast Cancer: Safety Analysis of MONARCH 2 and MONARCH 3. Oncologist 2021;26:e53-65.

21. Martin M, Hegg R, Kim SB, et al. Abemaciclib combined with adjuvant endocrine therapy in patients with high risk early breast cancer who received neoadjuvant chemotherapy (NAC). J Clin Oncol 2021;39:517.

22. Harbeck N, Johnston S, Fasching P, et al. Abstract PD201: High Ki-67 as a biomarker for identifying patients with high risk early breast cancer treated in monarchE. Cancer Res 2021. doi: 10.1158/1538-7445.SABCS20-PD2-01.

23. Yap Y, Kim S, Chiu J, et al. 48P Abemaciclib combined with adjuvant endocrine therapy in patients from Asia with high risk early breast cancer: monarchE. Ann Oncol 2021;32:S41-2.

24. Zhimin S, Zhang Q, Song CG, et al. Efficacy and safety analysis of Chinese patients in monarchE: Abemaciclib combined with adjuvant endocrine therapy for high risk HR+, HER2 - early breast cancer. J Clin Oncol 2021;39:522.

25. Mayer EL, Dueck AC, Martin M, et al. Palbociclib with adjuvant endocrine therapy in early breast cancer (PALLAS): interim analysis of a multicentre, open-label, randomised, phase 3 study. Lancet Oncol 2021;22:212-22.

26. O'Leary B, Finn RS, Turner NC. Treating cancer with selective CDK4/6 inhibitors. Nat Rev Clin Oncol 2016;13:417-30.

27. Hafner M, Mills CE, Subramanian K, et al. Multiomics Profiling Establishes the Polypharmacology of FDAApproved CDK4/6 Inhibitors and the Potential for Differential Clinical Activity. Cell Chem Biol 2019;26:1067-1080.e8.

28. Arimidex, Tamoxifen, Alone or in Combination (ATAC) Trialists' Group; Forbes JF, Cuzick J, et al. Effect of anastrozole and tamoxifen as adjuvant treatment for earlystage breast cancer: 100-month analysis of the ATAC trial. Lancet Oncol 2008;9:45-53.

29. Johnston SR, Yeo B. The optimal duration of adjuvant endocrine therapy for early stage breast cancer--with what drugs and for how long? Curr Oncol Rep 2014;16:358.

30. Harbeck N, Rastogi P, Martin M, et al. Adjuvant Abemaciclib Combined With Endocrine Therapy for High-Risk Early Breast Cancer: Updated Efficacy and Ki67 Analysis From the monarchE Study. Ann Oncol 2021. [Epub ahead of print].

31. Rugo H, O'Shaughnessy J, Song C, et al. Safety outcomes from monarchE: Phase 3 study of abemaciclib combined with endocrine therapy for the adjuvant treatment of HR+, HER-2-, node-positive, high risk, early breast cancer. Breast 2021;56:S23-4.

32. Hashimoto K, Shimomura A. Regarding the Addition of CDK4/6 Inhibitor to Postoperative Endocrine Therapy in Patients With HR-Positive HER2-Negative High-Risk Breast Cancer. J Clin Oncol 2021;39:1506-7.

33. Loibl S, Marmé F, Martin M, et al. Palbociclib for Residual High-Risk Invasive HR-Positive and HER2-Negative Early Breast Cancer-The Penelope-B Trial. J Clin Oncol 2021;39:1518-30.

34. Kawamura Y, Shimomura A, Shimizu C. Who Can Receive the Benefit of a Palbociclib Add-On in Patients With HR+HER2- Breast Cancer With Residual Disease After Neoadjuvant Chemotherapy? J Clin Oncol 2021;39:3089-90.

35. Kalinsky K, Barlow WE, Meric-Bernstam F, et al. Abstract GS3-00: First results from a phase III randomized clinical trial of standard adjuvant endocrine therapy (ET) +/chemotherapy (CT) in patients (pts) with 1-3 positive nodes, hormone receptor-positive (HR+) and HER2negative (HER2-) breast cancer (BC) with recurrence score (RS) <25: SWOG S1007 (RxPonder). Cancer Res 2021. doi: 10.1158/1538-7445.SABCS20-GS3-00

36. Condorelli R, Spring L, O'Shaughnessy J, et al. Polyclonal RB1 mutations and acquired resistance to CDK 4/6 inhibitors in patients with metastatic breast cancer. Ann Oncol 2018;29:640-5.

37. Yang $\mathrm{C}, \mathrm{Li} Z$, Bhatt $\mathrm{T}$, et al. Acquired CDK6 amplification promotes breast cancer resistance to CDK4/6 inhibitors and loss of ER signaling and dependence. Oncogene 2017;36:2255-64.

38. Turner NC, Slamon DJ, Ro J, et al. Overall Survival with Palbociclib and Fulvestrant in Advanced Breast Cancer. N Engl J Med 2018;379:1926-36. 
39. Dimitrakopoulos FI, Kottorou A, Tzezou A. Endocrine resistance and epigenetic reprogramming in estrogen receptor positive breast cancer. Cancer Lett 2021;517:55-65.

40. National_Comprehensive_Cancer_Network. National Comprehensive Cancer Network guideline. Available online: https://www.nccn.org/home

41. Hurvitz SA, Martin M, Press MF, et al. Potent Cell-Cycle Inhibition and Upregulation of Immune Response with Abemaciclib and Anastrozole in neoMONARCH, Phase II Neoadjuvant Study in HR+/HER2- Breast Cancer. Clin Cancer Res 2020;26:566-80.

42. Johnston S, Puhalla S, Wheatley D, et al. Randomized Phase II Study Evaluating Palbociclib in Addition to Letrozole as Neoadjuvant Therapy in Estrogen ReceptorPositive Early Breast Cancer: PALLET Trial. J Clin Oncol 2019;37:178-89.

doi: $10.21037 /$ tbcr-21-27

Cite this article as: Takahashi N, Shimizu C, Shimomura A, Toi M. Role of abemaciclib in primary breast cancer: a narrative review of MonarchE. Transl Breast Cancer Res 2022;3:3.
43. Alba E, Calvo L, Albanell J, et al. Chemotherapy (CT) and hormonotherapy (HT) as neoadjuvant treatment in luminal breast cancer patients: results from the GEICAM/2006-03, a multicenter, randomized, phase-II study. Ann Oncol 2012;23:3069-74.

44. Spring LM, Gupta A, Reynolds KL, et al. Neoadjuvant Endocrine Therapy for Estrogen Receptor-Positive Breast Cancer: A Systematic Review and Meta-analysis. JAMA Oncol 2016;2:1477-86.

45. Spring LM, Wander SA, Andre F, et al. Cyclin-dependent kinase 4 and 6 inhibitors for hormone receptorpositive breast cancer: past, present, and future. Lancet 2020;395:817-27.

46. Tutt ANJ, Garber JE, Kaufman B, et al. Adjuvant Olaparib for Patients with BRCA1- or BRCA2-Mutated Breast Cancer. N Engl J Med 2021;384:2394-405. 